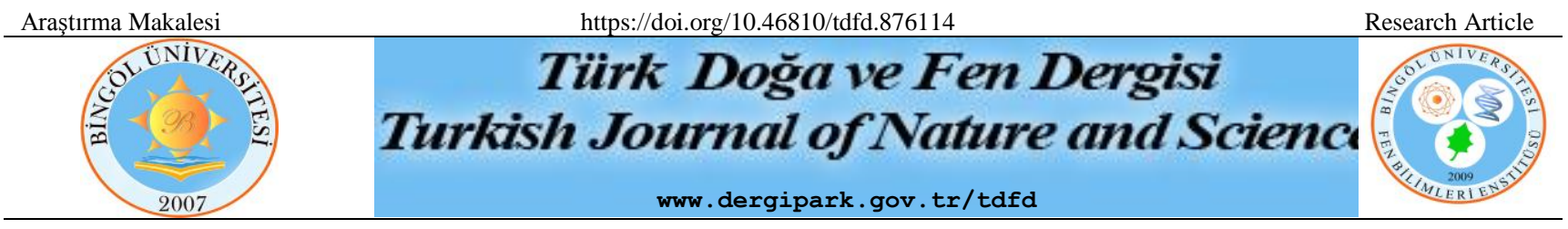

\title{
Metoksi Sübstitüe Kalkon İçeren Metalliftalosiyanin Bileşiklerinin Sentezi ve Karakterizasyonu
}

\author{
Furkan Özen ${ }^{1 *}$ \\ Dalı, 07058, Antalya, Türkiye \\ Furkan Özen ORCID No:0000-0003-1178-1167 \\ *Sorumlu yazar: furkanozen@akdeniz.edu.tr
}

Akdeniz Üniversitesi, Eğitim Fakültesi, Matematik ve Fen Bilimleri Eğitimi Bölümü, Fen Bilgisi Eğitimi Ana Bilim

(Alınış: 08.02.2021, Kabul: 05.03.2021, Online Yayınlanma: 25.06.2021)

Anahtar Kelimeler

Ftalosiyanin, metalliftalosiyanin, kalkon
Öz: $\mathrm{Bu}$ çalışmada, öncelikle 4-hidroksifenil-3-(4-metoksifenil) kalkon (1) bileşiği sentezlendi. Daha sonra 1 bileşiği 4-nitroftalonitrille reaksiyona sokularak 4-(4-(3-(4metoksifenil)akriloil)fenoksi)ftalonitril (2) bileşiği sentezlendi. 2 bileşiğinden yola çıkarak DBU katalizörlüğünde metalli ftalosiyanin bileşikleri elde edildi. Elde edilen yeni metalli ftalosiyanin bileşikleri DCM, THF, DMF ve DMSO gibi yaygın olarak kullanılan çözücülerde kolaylıkla çözünebilmektedir. Ftalosiyanin bileşiklerinin genellikle çözünürlüğü az olduğu için çok az çözücüde çözünmekte bir kısmı ise hiçbir çözücüde dahi çözünmemektedir. Sentezlenmiş olan bu metalli ftalosiyanin bileşiklerinin yukarıda belirtilen yaygın çözücülerde çözünmesi geniş kullanım ve uygulama alanı sağlayabilir. Sentez sonucu elde edilen bileşiklerin karakterizasyonu FT-IR, ${ }^{1} \mathrm{H}-\mathrm{NMR},{ }^{13} \mathrm{C}-\mathrm{NMR}$, UVVis ve MALDİ-TOF gibi spektroskopik yöntemler kullanılarak aydınlatılmıştır.

\section{Synthesis and Characterization of Metallyphthalocyanine Compounds Containing Methoxy Substituted Chalcone}

Keywords Phthalocyanin, metallophthalocyani ne, chalcone.

\begin{abstract}
This was firstly synthesized compound 4-hydroxyphenyl-3-(4methoxyphenyl)chalcone (1). Then, compound 1 was reacted with 4-nitrophthalonitrile and 4-(4-(3-(4-methoxyphenyl) acryloyl)phenoxy)phthalonitrile (2) compound was synthesized. DBU catalyzed metalic phthalocyanine compounds were obtained starting from the 2 compound. The new metal phthalocyanine compounds obtained can be dissolved in commonly used solvents such as DMSO, DMF, DCM and THF. Since phthalocyanine compounds are generally very poorly soluble, they dissolve in very little solvent and some of them do not dissolve even in any solvent. The dissolution of these synthesized phthalocyanine compounds in the above-mentioned common solvents can provide wide use and application. The characterization of compounds obtained as a result of the synthesis was illuminated by using spectroscopic methods such as FT-IR, ${ }^{1} \mathrm{H}-\mathrm{NMR},{ }^{13} \mathrm{C}-\mathrm{NMR}$, UV-Vis and MALDI-TOF.
\end{abstract}

\section{GíRiş}

Ftalosiyaninler kimyasal ve termal karalılığa sahip moleküller olup, makrosiklik halkada $18-\pi$ elektronu bulunan ve 400-700 nm arasında şiddetli absorpsiyon veren bileşiklerdir. Ftalosiyanin halkasına çeşitli sübstitüenler ve kordinasyon boşluğuna farklı metallerin katılmasıyla değişik özellikler kazandırılabilir [1]. Sahip olukları bu özelliklerinden dolayı teknolojik ürün sınıfına girmektedirler. Ftalosiyanin bileşikleri gaz sensör cihazlarda algılayıcı olarak [2], pigmentler ve boyalar [3], kimyasal sensörler [4], fotodinamik terapi duyarlılaştırıcılar [5], fotovoltaik malzemeler [6], elektrokromik malzemeler [7], elektronik cihaz bileşenleri [8], elektrokimyasal uygulamalar [9], enzim inhibisyonu [10,11], fotodiyot [12], fotokapasitör [13] gibi olarak kullanım alanları bulunmaktadır.

Kalkonlar flavonoid ailesine üye olup hem doğal hemde sentetik yolla elde edilebilen bileşikleridir. Endüstri alanında farklı uygulama alanlarına sahip olan 
kalkonların özelliklerinin belirlenmesi ve uygula alanlarının genişletilmesi sebebiyle güncel ve önemli bir konudur. Kalkonların uygulama alanlarından bazıları şunlardır; sitotoksik özellikler [14,15] elektrokromik özellikler [16], floresans özellikleri [17], termal ve dielektrik özellikler [18, 20] antimikrobial aktivite [21], anti-HIV aktivite [22,23], antibakterial activite [24], anti-inflammatory [25,26], anti-kanser aktiviteleri [27,28], DNA-bağlanma [29] ve enzim inhibisyonu $[30,31]$ gibi uygulama alanları bulunmaktadır.

Literatür çalışmalarından da görüldüğü gibi ftalosiyanin bileşiklerinin birçok kullanım alanı bulunmakta. Fakat ftalosiyanin bileşiklerinin dejavantanjlarından biri çözünürlük problemi bulunmaktadır. Bu çalışmanın en yenilikçi yönü kullanılan ftalosiyanin bileşiklerinin yaygın çözücülerde çözünebilen metalli ftalosiayanin bileşikleri sentezlemeye çalışarak daha geniş kullanım ve uygulama alanı sağlayan bileşikler elde edilmeye çalışılmıştır.

\section{MATERYAL VE METOT}

4-hidroksiasetofenon, 4-metoksibenzaldehit, 4nitroftalonitril, $\quad$ sodyumhidroksit $\quad(\mathrm{NaOH})$, potasyumkarbonat $\left(\mathrm{K}_{2} \mathrm{CO}_{3}\right)$, 1,8-diazabisiklo[5.4.0]undek-7-en (DBU), $\mathrm{Zn}(\mathrm{OAc})_{2} \cdot 2 \mathrm{H}_{2} \mathrm{O}$ ve $\mathrm{Co}(\mathrm{OAc})_{2} \cdot 4 \mathrm{H}_{2} \mathrm{O}$ kimyasallar satın alındı. Kullanılan çözücüler saflaştırma yöntemleri kullanılarak saflaştırıldı.

FT-IR spektrumları için ANTI UNICOM-Mattson 1000 FTIR spektroskopisi kullanıldı. Ultraviyole-visible spektroskopisi: UNICOM UV-2 spektroskopisi kullanıldı. NMR spektrumları $300 \mathrm{MHz}$ Bruker NMR spektrometresi ile ölçülmüştür. NMR analizi için $500 \mu \mathrm{L}$ döteryum çözücülerinde (d-kloroform) yaklaşık $20 \mathrm{mg}$ numune çözündürüldü.<smiles>COc1ccc(C=O)cc1</smiles><smiles>COc1ccc(/C=C/C(=O)c2ccc(Oc3ccc(/C=C/C(=O)c4ccc(Oc5ccc(C(=O)/C=C/c6ccc(OC)cc6)cc5)cc4)cc3)cc2)cc1</smiles> 


\subsection{4-hidroksifenil-3-(4-metoksifenil)kalkon'un (1) Sentezi ve Karakterizasyonu}

Kalkon bileşiği (1) literatüre göre sentezlendi [32,33]. $100 \mathrm{~mL}$ 'lik bir balonda $1 \mathrm{~g}$ (7.34 mmol) 4-hidroksi asetofenon $20 \mathrm{~mL}$ etanolde çözündü ve üzerine $3 \mathrm{~mL}$ $\% 30 \mathrm{NaOH}$ çözeltisi ilave edip $30 \mathrm{dk}$. oda sıcaklığında karıştırıldı. Daha sonra etanolde çözünen $1 \mathrm{~g}$ (7.34 mmol) 4-hidroksibenzaldehit buz altında damla damla reaksiyon ortamina ilave edildi. 24 saat boyunca oda sıcaklığında karıştırıldı. Reaksiyon durdurulduktan sonra buzlu suda son $\mathrm{pH} 6$ olacak şekilde çöktürüldü. Bol suyla yıkanıp kurutuldu. $1.1 \mathrm{~g}$ sarı katı madde elde edildi. Verim: \% 59. Molekül ağırlığı $254.29 \mathrm{~g} / \mathrm{mol}$. FTIR max/cm $\mathrm{cm}^{-1}: 3391 \mathrm{~cm}^{-1}(\mathrm{OH}) ; 3047 \mathrm{~cm}^{-1}(\mathrm{Ar}-\mathrm{H}) ; 2975$ $\mathrm{cm}^{-1}$ (alifatik C-H); $1643 \mathrm{~cm}^{-1}(\mathrm{C}=\mathrm{O}) ; 1599,1592 \mathrm{~cm}^{-1}$ $(\mathrm{Ar}-\mathrm{C}=\mathrm{C}) ; 1035 \mathrm{~cm}^{-1}(\mathrm{C}-\mathrm{O}-\mathrm{C}) .{ }^{1} \mathrm{H}$ NMR $(300 \mathrm{MHz}$, $\left.\mathrm{CD}_{3} \mathrm{OH}\right): \delta$ ppm $7.88\left(1 \mathrm{H}, \mathrm{d}, \mathrm{H}^{6}\right), 7.84\left(2 \mathrm{H}, \mathrm{d}, \mathrm{H}^{10}\right), 7.54$ $\left(4 \mathrm{H}, \mathrm{d}, \mathrm{H}^{4}\right), 7.39\left(1 \mathrm{H}, \mathrm{d}, \mathrm{H}^{7}\right), 6.87\left(2 \mathrm{H}, \mathrm{d}, \mathrm{H}^{3}\right), 6.80(2 \mathrm{H}$, $\left.\mathrm{s}, \mathrm{H}^{11}\right), 3.76\left(3 \mathrm{H}, \mathrm{s}, \mathrm{H}^{1}\right)$ ve $3.67\left(1 \mathrm{H}, \mathrm{s}, \mathrm{H}^{13}\right) .{ }^{13} \mathrm{C}-\mathrm{NMR}$ (300 MHz, $\left.\mathrm{CD}_{3} \mathrm{OH}\right): 189.89 \mathrm{C}^{8}, 162.00 \mathrm{C}^{2}, 161.70 \mathrm{C}^{12}$, 144.22 $C^{6}, 131.23 C^{10}, 130.32 C^{4}, 130.18 C^{9}, 127.78 C^{5}$, $119.67 \mathrm{C}^{7}, 115.54 \mathrm{C}^{11}, 114.52 \mathrm{C}^{3}$ ve $55.53 \mathrm{C}^{1}$.

\subsection{4-(4-(3-(4-metoksifenil)akriloil)fenoksi)ftalonitril (2)'in Sentezi ve Karakterizasyonu}

50 mL'lik bir balonda $0.5 \mathrm{~g} \quad(2.88 \mathrm{mmol})$ 4nitroftalonitril $15 \mathrm{~mL}$ DMF'de çözüldü üzerine $0.73 \mathrm{~g}$
(2.88 mmol) 4-hidroksifenil-3-(4-metoksifenil) kalkon (1) ilave edildikten sonra $0.4 \mathrm{~g}(2.89 \mathrm{mmol}) \mathrm{K}_{2} \mathrm{CO}_{3}$ azar azar 1 saat zaman peryodunda ilave edildikten sonra reaksiyona azot atmosferinde ve $50{ }^{\circ} \mathrm{C}$ 'de 24 saat devam edildi. Reaksiyon sonunda çözelti buzlu suda çöktürüldü. Bol suyla yıkandıktan sonra kurutuldu. $0.9 \mathrm{~g}$ açı sarı madde elde edildi. Verim\% 82. Molekül ağırlığı: 380.40 g/mol. Erime noktasi: $176{ }^{\circ} \mathrm{C}$. FT-IR $\max / \mathrm{cm}^{-1}: 3099$ $\mathrm{cm}^{-1}(\mathrm{Ar}-\mathrm{H}) ; 3037 \mathrm{~cm}^{-1}$ (alifatik C-H); $2230 \mathrm{~cm}^{-1}$ $(\mathrm{C} \equiv \mathrm{N}) ; 1658 \mathrm{~cm}^{-1}(\mathrm{C}=\mathrm{O}) ; 1588,1561 \mathrm{~cm}^{-1}(\mathrm{C}=\mathrm{C}) ; 1028$ $\mathrm{cm}^{-1}$ (C-O-C). FT-IR spektrumunda $\mathrm{OH}$ grubuna ait pikin $3391 \mathrm{~cm}^{-1}$ de gözlememesi ve $\mathrm{CN}$ ait pikin 2230 $\mathrm{cm}^{-1}$ de gözlenmesi yapının oluştuğunu göstermektedir. ${ }^{1} \mathrm{H}$ NMR $\left(300 \mathrm{MHz}, \mathrm{CDCl}_{3}\right): \delta \mathrm{ppm} 8.11\left(2 \mathrm{H}, \mathrm{d}, \mathrm{H}^{4}\right)$, 7.84-7.79 $\left(2 \mathrm{H}, \mathrm{m}, \mathrm{H}^{7}\right.$ ve $\left.\mathrm{H}^{8}\right), 7.60\left(2 \mathrm{H}, \mathrm{d}, \mathrm{H}^{11}\right), 7.42$ $7.30\left(3 \mathrm{H}, \mathrm{m}, \mathrm{H}^{14}, \mathrm{H}^{17}, \mathrm{H}^{18}\right), 7.37\left(1 \mathrm{H}, \mathrm{d}, \mathrm{H}^{18}\right), 7.16(2 \mathrm{H}$, $\left.\mathrm{d}, \mathrm{H}^{10}\right), 6.93\left(2 \mathrm{H}, \mathrm{d}, \mathrm{H}^{3}\right)$, ve $3.86\left(3 \mathrm{H}, \mathrm{s}, \mathrm{OCH}_{3}\right) .{ }^{1} \mathrm{H}$ NMR spektrumunda $3.76 \mathrm{ppm}$ deki $\mathrm{OH}$ protonun ait pikin gözlenmemesi ve spektrumda aromatik bölde yeni piklerin gözlenmesi yapının oluştuğunu göstermektedir. ${ }^{13} \mathrm{C}-\mathrm{NMR}\left(300 \mathrm{MHz}, \mathrm{CDCl}_{3}\right.$ ): $188.99 \mathrm{C} 8,162.15 \mathrm{C}^{2}$, $160.87 \mathrm{C}^{12}, 157.43 \mathrm{C}^{13}, 145.63 \mathrm{C}^{6}, 136.42 \mathrm{C}^{17}, 135.85$ $\mathrm{C}^{10}, 131.44 \mathrm{C}^{9}, 130.64 \mathrm{C}^{4}, 127.55 \mathrm{C}^{5}, 122.61 \mathrm{C}^{18}, 122.42$ $\mathrm{C}^{14}, 120.37 \mathrm{C}^{11}, 119.17 \mathrm{C}^{7}, 118.10 \mathrm{C}^{15}, 115.45 \mathrm{C}^{19}$, $115.01 \mathrm{C}^{20}, 114.73 \mathrm{C}^{3}, 110.05 \mathrm{C}^{16}$ ve $55.71 \mathrm{C}^{1} .{ }^{13} \mathrm{C} \mathrm{NMR}$ spektrumunda $115.45 \mathrm{C}^{19}$ ve115.01 $\mathrm{C}^{20} \mathrm{CN}$ karbonlarına ve aromatik halka karbonlarına ait piklerin gözlenmesi yapının oluştuğunu göstermektedir.

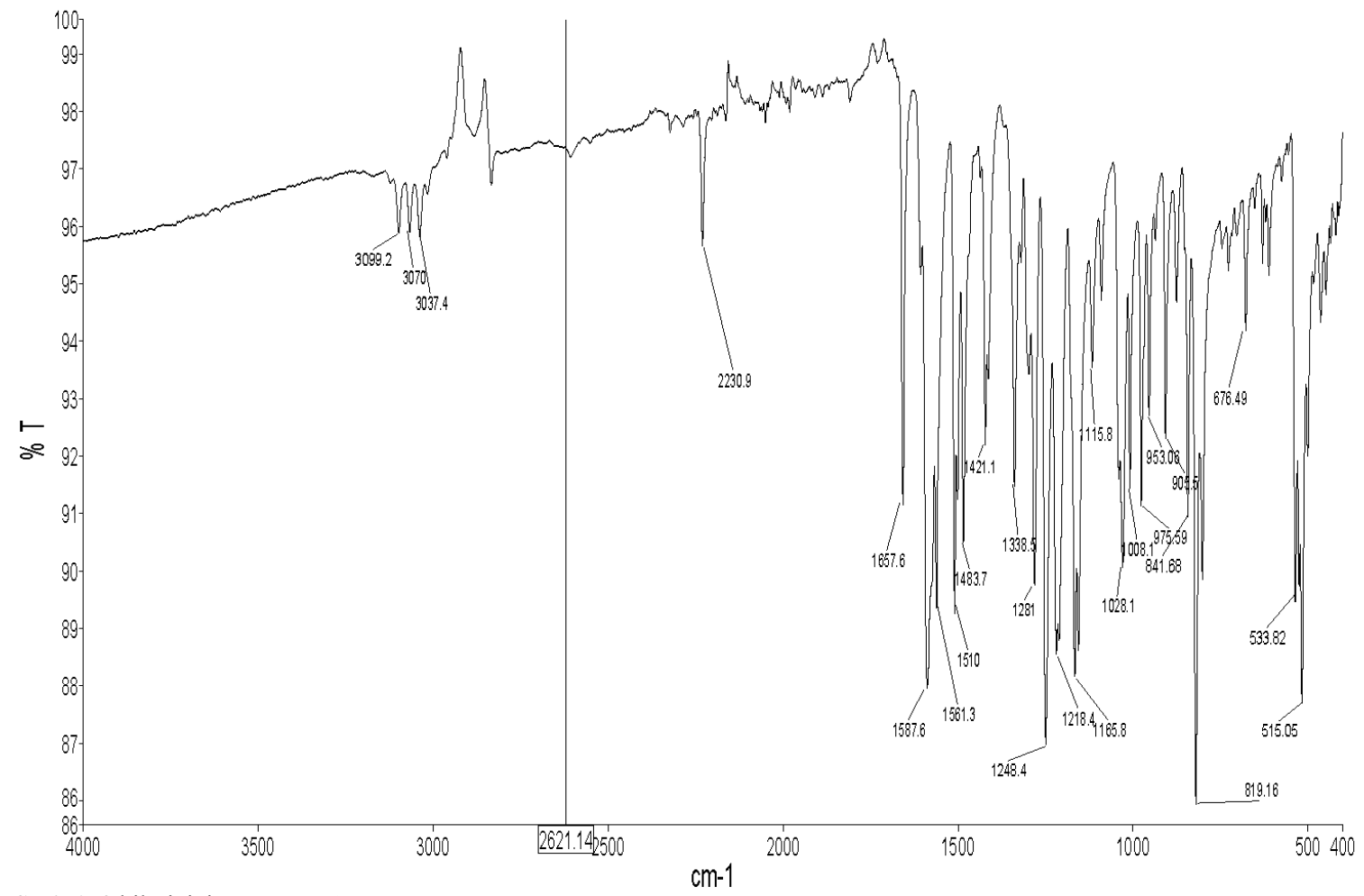

Şekil 1: 2 bileşiğinin FT-IR spektrumu (ATR) 

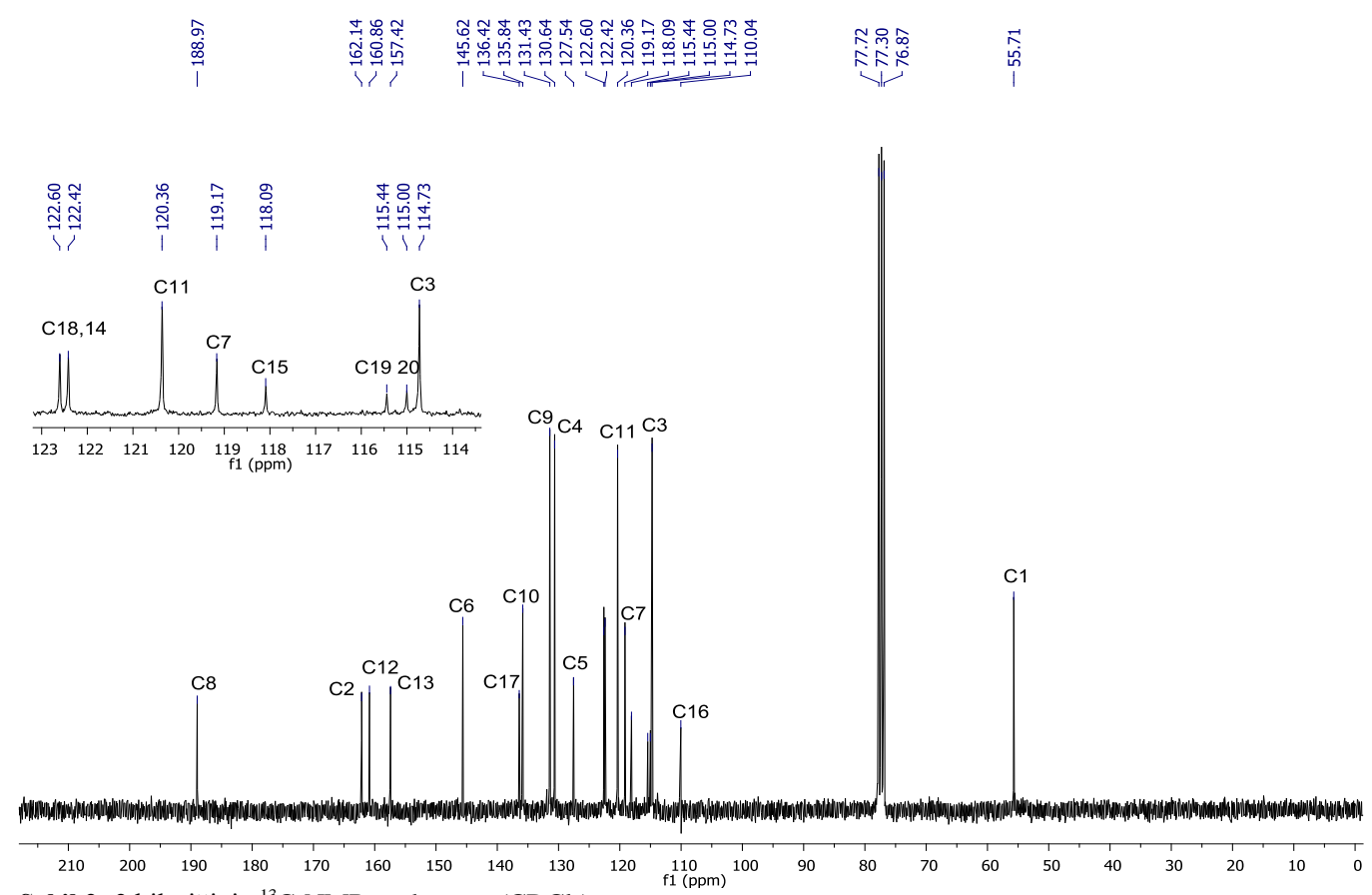

Şekil 2: 2 bileşiğininin ${ }^{13} \mathrm{C}-\mathrm{NMR}$ spektrumu $\left(\mathrm{CDCl}_{3}\right)$

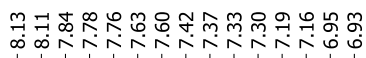

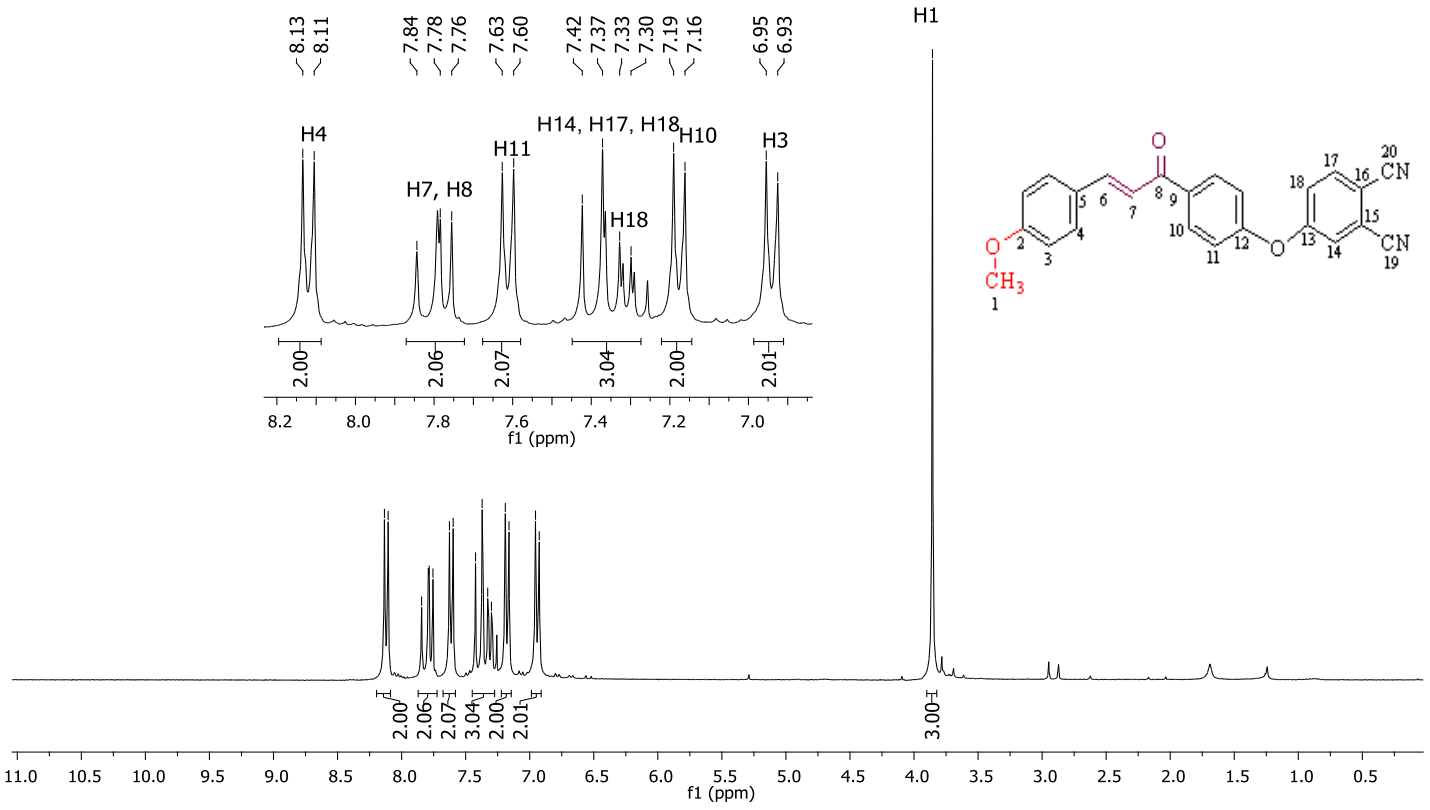

H1

Şekil 3: 2 bileşiğinin ${ }^{1} \mathrm{H}$ NMR spektrumu $\left(\mathrm{CDCl}_{3}\right)$

\section{3. Çinko (II) ftalosiyanin (2a)'nın Sentezi ve Karakterizasyonu}

$25 \mathrm{~mL}$ 'lik bir balona $0.2 \mathrm{~g}(0.52 \mathrm{mmol}) 2$ bileşiği $10 \mathrm{~mL}$ DMF'de çözündü üzerine $0.058 \mathrm{~g} \quad(1.05 \mathrm{mmol})$ $\mathrm{Zn}(\mathrm{OAc})_{2} \cdot 2 \mathrm{H}_{2} \mathrm{O}$ ilave edildikten sonra üzerine 2-3 damla 1,8-diazabisiklo[5.4.0]-undek-7-en (DBU) ilave edip azot ortamında, yağ banyosu altında, $130{ }^{\circ} \mathrm{C}$ 'de 24 saat karıştırıldı. Reaksiyon sonlandırıldıktan sonra buzlu suda çöktürüldü, süzüldü. Bol su ve sıcak alkolle yıkanıp kurutuldu. $0.1 \mathrm{~g}$ açık laciver madde elde edildi. Bu madde kolaylıkla DCM, THF, DMF ve DMSO da çözüne bilmektedir. Molekül ağırlığı 1586.99. Verim \%
46. Erime noktas $1>350{ }^{\circ} \mathrm{C}$. FT-IR $v_{\max }\left(\mathrm{cm}^{-1}\right): 3066 \mathrm{~cm}^{-1}$ $(\mathrm{Ar}-\mathrm{H}) ; 2948 \mathrm{~cm}^{-1}$ (alifatik C-H); $1652 \mathrm{~cm}^{-1}(\mathrm{C}=\mathrm{O})$; 1593, $1570(\mathrm{C}=\mathrm{N}, \mathrm{C}=\mathrm{C}) ; 1026 \mathrm{~cm}^{-1}(\mathrm{C}-\mathrm{O}-\mathrm{C})$. FT-IR spektrumunda $-\mathrm{CN}$ grubuna ait pikin gözlenmemesi yapının oluştuğunun en önemli kanıtıdır. UV-vis (DMF), $\lambda_{\max }, \mathrm{nm}: 678,613,360$. UV-vis spektrumunda Zn metali için 678, $613 \mathrm{~nm}$ de sinyallerin gözlenmesi yapının oluştuğunu göstermektedir. MALDI-TOF MS: $\mathrm{m} / \mathrm{z}[\mathrm{M}]^{+}$hesaplanan $\mathrm{C}_{96} \mathrm{H}_{64} \mathrm{~N}_{8} \mathrm{O}_{12} \mathrm{Zn}$ : 1586.99 ; bulunan $[\mathrm{M}+\mathrm{H}]^{+}$1585.15. MALDI-TOF MS spektrumunda teorik ve bulunan değerlerin hemen hemen aynı olması yapının oluştuğunu göstermektedir. 


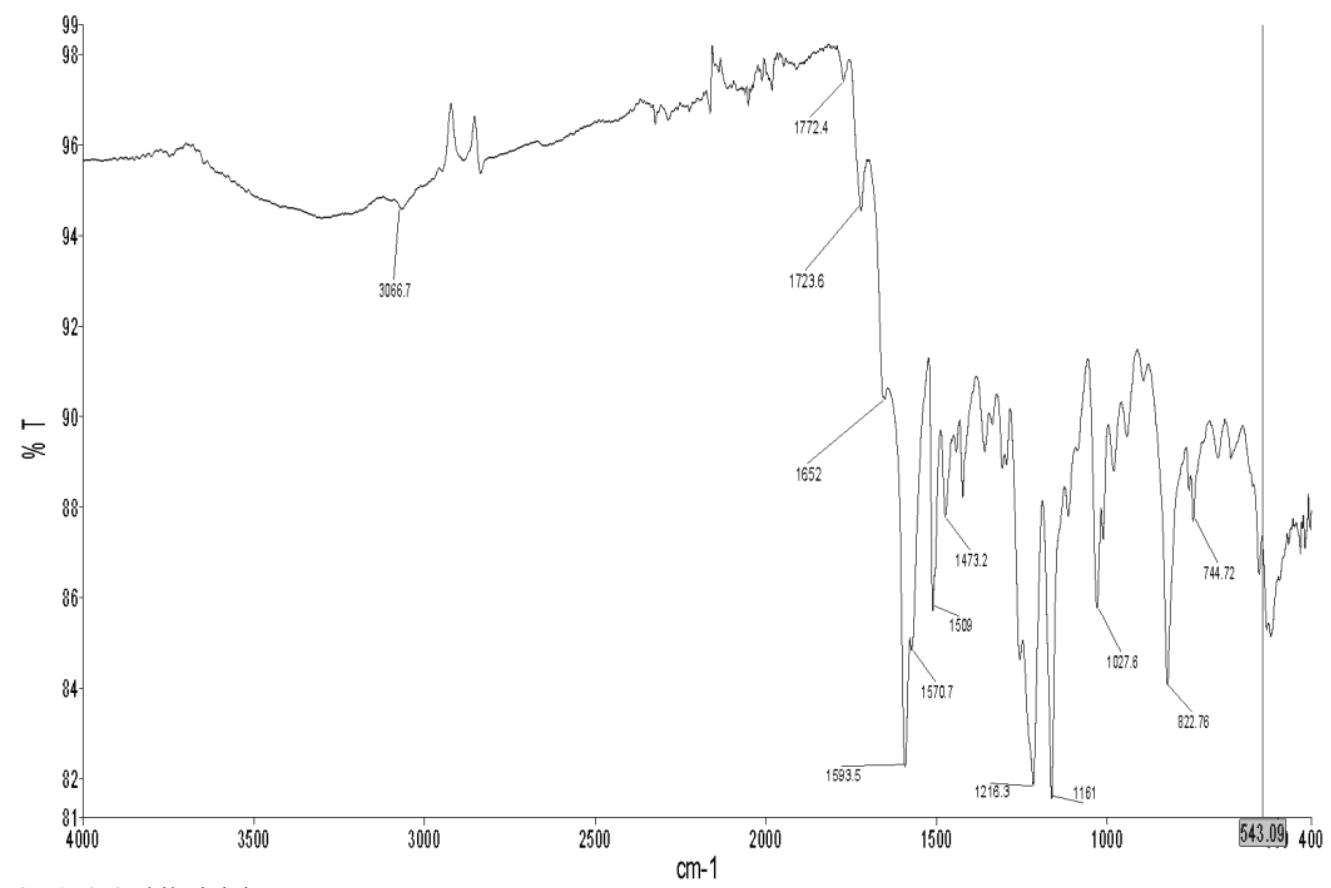

Şekil 4: 2a bileşiğinin FT-IR spektrumu (ATR)

\subsection{Cobalt (II) ftalosiyanin (2b)'nun Sentezi ve Karakterizasyonu}

$25 \mathrm{~mL}$ 'lik bir balona $0.2 \mathrm{~g}(0.52 \mathrm{mmol}) 2$ bileşiği $10 \mathrm{~mL}$ DMF'de çözündü üzerine $0.057 \mathrm{~g} \quad(1.05 \mathrm{mmol})$ $\mathrm{Co}(\mathrm{OAc})_{2} \cdot 4 \mathrm{H}_{2} \mathrm{O}$ ilave edildikten sonra üzerine 2-3 damla 1,8-diazabisiklo[5.4.0]-undek-7-en (DBU) ilave edip azot ortamında, yağ banyosu altında, $130{ }^{\circ} \mathrm{C}$ 'de 24 saat karıştırıldı. Reaksiyon sonlandırıldıktan sonra buzlu suda çöktürüldü, süzüldü. Bol su ve sıcak alkolle yıkanıp kurutuldu. $0.1 \mathrm{~g}$ açık laciver madde elde edildi. Bu madde kolaylıkla DCM, THF, DMF ve DMSO da çözüne bilmektedir. Molekül ağırlığı 1580.55. Verim \%
43. Erime noktas $1>350{ }^{\circ} \mathrm{C}$. FT-IR $v_{\max }\left(\mathrm{cm}^{-1}\right): 3066 \mathrm{~cm}^{-1}$ (Ar-H); $2948 \mathrm{~cm}^{-1}$ (alifatik C-H); $1651 \mathrm{~cm}^{-1}(\mathrm{C}=\mathrm{O})$; 1593, $1570(\mathrm{C}=\mathrm{N}, \mathrm{C}=\mathrm{C}) ; 1026 \mathrm{~cm}^{-1}(\mathrm{C}-\mathrm{O}-\mathrm{C})$. FT-IR spektrumunda $-\mathrm{CN}$ grubuna ait pikin gözlenmemesi yapının oluştuğunun en önemli kanıtıdır. UV-vis (DMF), $\lambda_{\max }, \mathrm{nm}: 690,623,353$. UV-Vis spektrumunda Co metali için 690, $623 \mathrm{~nm}$ deki sinyallerin gözlenmesi yapının oluştuğunu göstermektedir. MALDI-TOF MS: $\mathrm{m} / \mathrm{z}[\mathrm{M}]^{+}$hesaplanan $\mathrm{C}_{96} \mathrm{H}_{64} \mathrm{~N}_{8} \mathrm{O}_{12} \mathrm{Co}: 1580.55$; bulunan $[\mathrm{M}+\mathrm{H}]^{+}$1579.50. MALDI-TOF MS spektrumunda teorik ve bulunan değerlerin hemen hemen aynı olması yapının oluştuğunu göstermektedir.

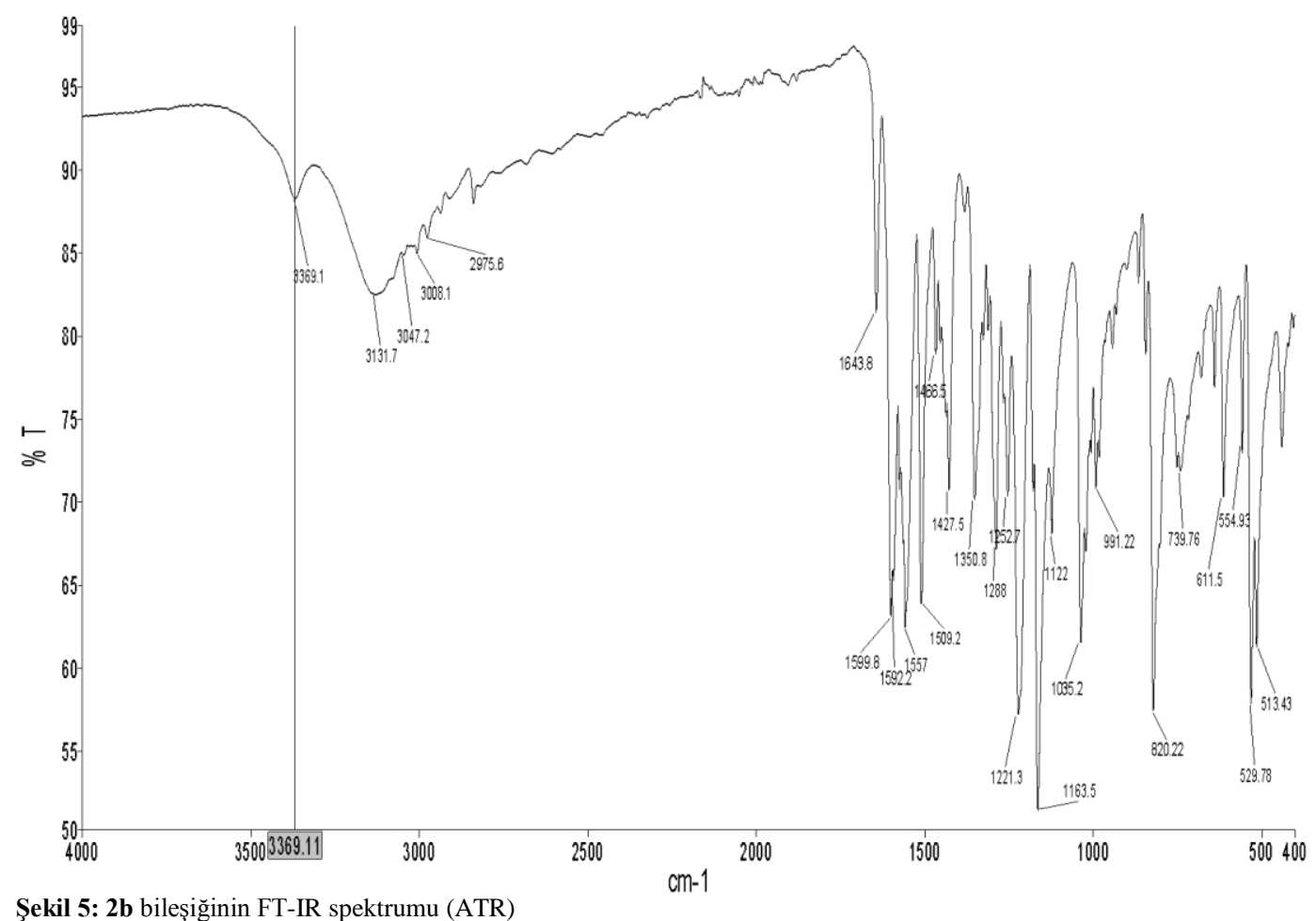




\section{SONUÇ}

1. $\mathrm{Bu}$ deneysel kısımda belirtildiği gibi 4hidroksifenil-3-(4-metoksifenil)kalkon (1) literatüre göre $[27,28]$ sentezlendikten sonra 4-nitroftalonitril ile tepkimeye sokularak 4-(4-(3-(4-metoksifenil)akriloil)fenoksi)ftalonitril (2) bileşiği sentezlendi. 2 bileşiği $\mathrm{Zn}$ ve Co metal tuzları kullanılarak metoksi sübstitüe metalli ftalosiyanin bileşikleri sentezlendi. Sentezlenen yapılar FT-IR, ${ }^{1} \mathrm{H}-\mathrm{NMR}$ ve ${ }^{13} \mathrm{C}-\mathrm{NMR}$, UV-Vis ve MALDİ MS teknikleri kullanılarak karakterize edildi. Tüm bu karakterizasyonları çalışmaları sonucu yapıları aydınlatılan yeni metoksi sübstitüe metalli ftalosiyanin bileşikleri sentezlendi.

2. Metalli ftalosiyanin bileşiklerinin oluştuğunun en belirgin kanıtı FT-IR spektrumunda $2230 \mathrm{~cm}^{-1}$ deki $\mathrm{CN}$ pikin gözlenmemesi ve UV-Vis spektrumunda $\mathrm{Zn}$ metali için 678, $613 \mathrm{~nm}$ de ve Co metali için 690, 623 nm deki sinyallerin gözlenmesi yapının oluştuğunu göstermektedir. Ayrıca kütle spektrumlarındaki moleküler iyon pikleri de önerilen molekül formüllerini desteklemektedir.

3. Literatürde sentezlenen ftalosiyanin bileşiklerinden farklı olarak sentezlenen metalli ftalosiyanin bileşikleri birçok çözücüde çözünmesi geniş kullanım ve uygulama alanı sağlayabilir.

4. Bu çalışmada elde edilen metalli ftalosiyanin bileşiklerinin verimleri literatürde sentezlenen bileşiklere göre daha yüksektir [12].

5. $\mathrm{Bu}$ çalışmada metalsiz, $\mathrm{Ni}$ ve $\mathrm{Cu}$ metalliftalosiyanin bileşikleri aynı reaksiyon şartlarında elde edilememiştir.

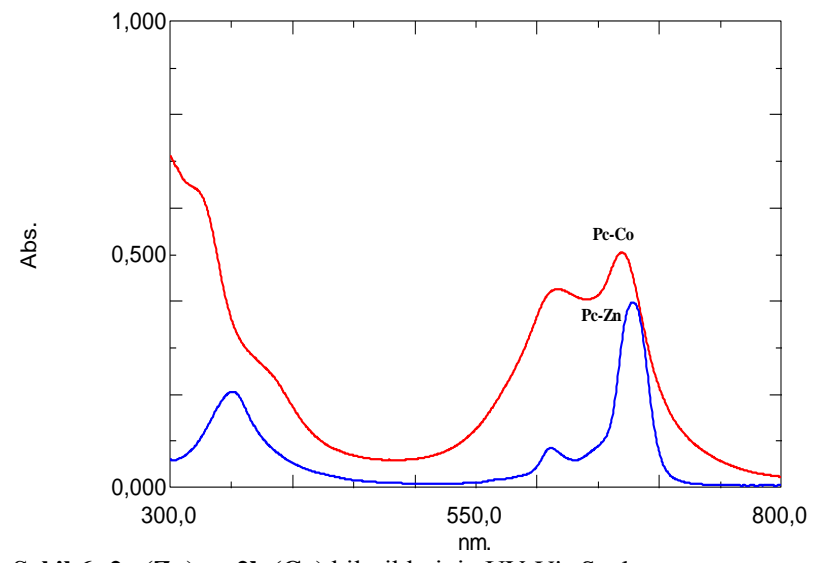

Şekil 6: 2a (Zn) ve 2b (Co) bileşiklerinin UV-Vis Spektrumu

\section{KAYNAKLAR}

[1] Kadish, K. M., Smith, K. M., \& Guilard, R.. Handbook of Porphyrin Science: with Applications to Chemistry, Physics, Materials Science, Engineering, Biology and Medicine (Volumes 1-5). World Scientific: Singapore, 2010, 1-35.

[2] Yazıcı, A, Dalbul, N, Altındal, A, Salih, B, Bekaroğlu, Ö. Ethanol sensing property of novel phthalocyanines substituted with 3,4-dihydroxy3-cyclobuten-1,2-dione. Sensors and Actuators B. 2014;202:14-22.

[3] Mert Sevim A, Ilgün C, Gül A. Preparation of heterogeneous phthalocyanine catalysts by cotton fabric dyeing. Dyes and Pigments. 2011;89:162168.

[4] Yazıcı A, Dalbul N, Altındal A, Salih B, Bekaroğlu Ö. Ethanol sensing property of novel phthalocyanines substituted with 3,4-dihydroxy3-cyclobuten-1,2-dione. Sensors and Actuators B. 2014;202:14-22.

[5] Zheng BY, Zhang HP, Ke MR, Huang JD. Synthesis and antifungal photodynamic activities of a series of novel zinc (II) phthalocyanines substituted with piperazinyl moieties. Dyes and Pigments. 2013;99:185-191.

[6] Soylu M, Ocaya R, Tuncer H, Al-Ghamdi AA, Dere A, Sari DC, Yakuphanoglu F. Analysis of photovoltaic behavior of Si-based junctions containing novel graphene oxide/nickel (II) phthalocyanine composite films. Microelectronic Engineering. 2016;154:53-61.

[7] Sena P, Dumludag F, Salih B, Özkaya AR, Bekaroglu Ö. Synthesis and electrochemical, electrochromic and electrical properties of novel s-triazine bridged trinuclear $\mathrm{Zn}$ (II), $\mathrm{Cu}$ (II) and $\mathrm{Lu}$ (III) and a tris double-decker $\mathrm{Lu}$ (III) phthalocyanines. Synthetic Metals. 2011;161:1245-1254.

[8] Grobosch M, Schmidt C, Kraus R, Knupfer M. Electronic properties of transition metal phthalocyanines: The impact of the central metal atom $\left(\mathrm{d}^{5}-\mathrm{d}^{10}\right)$. Organic Electronics. 2010;11:1483-1488.

[9] Dinçer HA, Koca A, Gül A, Koçak MB. Novel phthalocyanines bearing both quaternizable and bulky substituents. Dyes and Pigments. 2008;76:825-831.

[10] Kantar C, Mavi V, Baltas N, Islamoğlu F, Şaşmaz S. Novel zinc (II) phthalocyanines bearing azocontaining schiff base: Determination of $\mathrm{pKa}$ values, absorption, emission, enzyme inhibition and photochemical properties. Journal of Molecular Structure. 2016;1122:88-99.

[11] Kantar GK, Baltaş N, Menteșe E, Şaşmaz S. Microwave-assisted synthesis and investigation of xanthine oxidase inhibition of new phthalonitrile and phthalocyanines containing morpholino substituted 1,2,4-triazole-3-one. Journal of Organometallic Chemistry. 2015;787:8-13.

[12] Demirol, M., Sirka, L., Çalışkan, E., Biryan, F., Koran, K., Görgülü, A. O., \& Yakuphanoğlu, F.. Synthesis and photodiode properties of chalcone substituted metallo-phthalocyanine. Journal of Molecular Structure. 2020; 1219, 128571.

[13] Tataroglu, A., Koran, K., Çaliskan, E., AlSehemi, A. G., Görgülü, A. O., Al-Ghamdi, A., \& Yakuphanoglu, F. Metallo-Phthalocyanines Based Photocapacitors. Silicon. 2019;11(3), 1275-1286. 
[14] Koran, K., Tekin, Ç., Biryan, F., Tekin, S., Sandal, S., \& Görgülü, A. O. Synthesis, structural and thermal characterizations, dielectric properties and in vitro cytotoxic activities of new 2,2,4,4-tetra(4'-oxy-substituted-chalcone)-6,6diphenylcyclotri-phosphazene derivatives. Medicinal Chemistry Research. 2017; 26(5), 962974.

[15] Koran, K., Tekin, Ç., Çalışkan, E., Tekin, S., Sandal, S., \& Görgülü, A. O. Synthesis, structural and thermal characterizations and in vitro cytotoxic activities of new cyclotriphosphazene derivatives. Phosphorus, Sulfur, and Silicon and the Related Elements. 2017;192(9), 1002-1011.

[16] Jin H, Li X, Tan T, Wang S, Xiao Y, Tian J. Electrochromic properties of novel chalcones containing triphenylamine moiety. Dyes and Pigments. 2014;106:154-160.

[17] Patil PS, Maidur SR, 1 Rao SV, Dharmaprakash S.M. Crystalline perfection, third-order nonlinear optical properties and optical limiting studies of 3, 4-Dimethoxy-4'-methoxychalcone single crystal. Optics \& Laser Technology. 2016;81:7076.

[18] Koran K, Özen F, Biryan F, Demirelli K, Görgülü AO. $\mathrm{Eu}^{+3}$-doped chalcone substituted cyclotriphosphazenes: Synthesis, characterizations, thermal and dielectrical properties. Inorganica Chimica Acta. 2016;450:162-169.

[19] Koran K, Ozen F, Torğut G, Pihtılı G, Cil E, Gorgulu AO, Arslan M. Synthesis, characterization and dielectric properties of phosphazenes containing chalcones. Polyhedron. 2014;79:213-220.

[20] Koran, K. Structural, chemical and electrical characterization of organocyclotri-phosphazene derivatives and their graphene-based composites. Journal of Molecular Structure, 2019;1179, 224232.

[21] Gupta R, Chaudhary RP. Synthesis, antimicrobial and DFT studies of novel fused thiazolopyrimidine derivatives. Heterocycl Commun. 2013;19:207-214.

[22] Cole AL, Hossain S, Cole AM, Phanstiel O. Synthesis and bioevaluation of substituted chalcones, coumaranones and other flavonoids as anti-HIV agents. Bioorganic \& Medicinal Chemistry. 2016;24:2768-2776.

[23] Hu G, Li X, Zhang X, Li Y, Ma L, Yang LM, Liu G, Li W, Huang J, Shen X, Hu L, Zheng YT, Tang $Y$. Discovery of 1nhibitors to block interactions of HIV-1 integrase with human LEDGF/p75 via structure-based virtual screening and bioassays. J Med Chem. 2012;55:10108-10117.

[24] Rudrapal M, Satyanandam RS, Swaroopini TS, Lakshmi TN, Jaha SK, Zaheera S. Synthesis and antibacterial activity of some new hydrazones. Med Chem Res. 2013;22:2840-2846.

[25] Lin CT, Kumar KJS, Tseng YH, Wang ZJ, Pan MY, X1ao JH, Chien SC, Wang SY. Antiinflammatory activity of flavokawain $b$ from alpinia pricei hayata. J Agric Food Chem. 2009;57:6060-6065

[26] Li YY, Huang SS, Lee MM, Deng JS, Huang GJ. Anti-inflammatory activities of cardamonin from Alpinia katsumadai through heme oxygenase-1 induction and inhibition of NF-KB and MAPK signaling pathway in the carrageenan-induced paw edema. International Immunopharmacology. 2015;25:332-339.

[27] Gorgulu AO, Koran K, Ozen F, Tekin S, Sandal S. Synthesis, structural characterization and anticarcinogenic activityof new cyclotriphosphazenes containing dioxybiphenyl and chalcone groups. Journal of Molecular Structure. 2015;1087:1-10.

[28] Lee Y, Kim BS, Ahn S, Koh D, Lee YH, Shin SY, Lim Y. Anticancer and structure-activity relationship evaluation of 3-(naphthalen-2-yl)N,5-diphenyl-pyrazoline-1-carbothioamide analogs of chalcone. Bioorganic Chemistry. 2016;68:166-176.

[29] Kamal A, Shankaraiah N, Prabhakar S, Reddy CR, Markandeya N, Reddy KL, Devaiah V. Solid-phase synthesis of new pyrrolobenzodiazepine-chalcone conjugates: DNA-binding affinity and anticancer activity. Bioorganic \& Medicinal Chemistry Letters. 2008;18:2434-2439.

[30] Li H, Chen Y, Zhang B, Niu X, Song M, Luo Z, Lu G, Liu B, Zhao X, Wang J, Deng X. Inhibition of sortase A by chalcone prevents listeria monocytogenes infection. Biochemical Pharmacology. 2016;106:19-29.

[31] Wang H, Wang Y, Chen Z, Chan FL, Leung LK. Hydroxychalcones exhibit differential effects on XRE Transactivation. Toxicology. 2005;207:303-313.

[32] B.S. Funiss, A.J. Hannford, P.W.G. Smith, A.R. Tatchell, Vogel's Textbook of Practical Org. Chem. 5th ed., Longman, London, 2004. p10321035 .

[33] Shakir M, Azam M, Parveen S, Khan A U, Firdaus F. Synthesis and spectroscopic studies on complexes of N,N'-bis-(2pyridinecarboxaldimine)-1,8-diaminonaphthalene (L); DNA binding studies on $\mathrm{Cu}$ (II) complex.Spectrochim Acta A. 2009;71:18511856. 